immerged weights of the cuts (specific gravity and tissular composition). The cutting criteria thus determine an amplitude of variation between $5^{2}$ and $57 \mathrm{p}$. Ioo of lean cuts, between I9 and $13 \mathrm{p}$. Ioo fat cuts. The discrimination threshold of the point (or I p. roo) of this variation was directly related to the variation of the carcass net weight. As the yield was higher in fat pigs, the fat masses were underestimated ; inversely, the lean masses of the lean pigs were overestimated by these modes of expression that did not correspond with the carcass fattening state differences. Castration of male pigs tended to equalize the differences between the 3 genetic types, whereas the discrimination was clearly established with female pig carcasses. The criteria classically used to estimate the carcasses generally appeared to be insufficient for the selection of lean pigs (at the moment of progeny-testing). Further development of the methods used to measure the fattening state of the carcasses would be necessary for determining the heterosis effect as well as for adapting the feeding of the animals to the different levels of genetic potentiality.

\title{
ÉTUDE DU PROFIL DE LA COUCHE DE LARD DU POLC MALE ENTIER DE RACE « LARGE-WHITE » DE 80 ET $100 \mathrm{KG}$
}

\author{
B.-L. DUMONT \\ Laboratoire de Recherches sur la Viande, \\ Centre national de Recherches zootechniques, I. N.R. A., \\ 78350 Jouy en Josas
}

\section{RÉSUMMÉ}

La couche de graisse sous-cutanéc au niveau de la colonne vertébrale (lard dorsal) de porcs mâles de race Large White de 80 et Ioo $\mathrm{kg}$ de poids vif montre un maximum au niveau de la seconde côte et un minimum en avant de la dernière côte. Les profils de lard dorsal sont semblables chez les animaux de 80 et Ioo kg, mais l'augmentation d'épaisseur, entre ces deux stades, n'est significative qu'au niveau de garrot et de l'attache du rein. L'augmentation d'épaisseur moyenne du lard (9 p. roo) et celle de la longueur de la carcasse ( $6 \mathrm{p}$. тоo) n'explique que très partiellement l'accroissement de la "bardière " (3o p. Ioo environ). Les relations entre l'épaisseur du lard aux divers niveaux et le poids de la "bardière " sont très différents chez les mâles castrés et les entiers. Pour ces derniers, le milieu du rein est la zone où les corrélations sont les plus fortes. Les méthodes d'appréciation de l'adiposité à partir de l'épaisseur de lard en usage pour les autres types sexuels ne peuvent être transposées aux mâles entiers.

\section{SUMMARY}

\section{STUDY OF BACKFAT THICKNESS ALONG THE BACKBONE OF NON CASTRATED LARGE WHITE MALE PIGS OF 80 AND IOO KG LIVE WEIGHT}

Backfat thickness in Large White male pigs of 80 and $100 \mathrm{~kg}$ live weight was maximum at second rib level and minimum in front of the last rib. The backfat profiles were similar in animals of 80 and $100 \mathrm{~kg}$, but the increase in thickness, between these two stages, was only significant 
in two areas: withers and junction of thoracic and lumbar vertebrae. The increase of the mean backfat thickness (9 p. I00) and that of the carcass length (6 p. 100) only partially explain the backfat weight gain, about $30 \mathrm{p}$. Ioo, between 80 and $100 \mathrm{~kg}$. The relationship between backfat thickness at different levels and backfat weight were very different in castrated and non castrated male pigs. As for the latter correlations were highest in the middle of the loin. Methods of estimating fatness from backfat thickness used in the other sexual types cannot be transposed to non castrated males.

\title{
COMPARAISONS DES CARACTËES ANATOMIQUES DE LA RÉGION DORSO-LOMBAIRE DES PORCS MALES ENTIERS « LARGE-WHITE » DE 80 ET 100 KG
}

\author{
B.-L. DUMONT et B. DESMOULIN* \\ Laboratoire de Recherches sur la Viande, \\ * Station de Recherches sur l'Élevage des Porcs, \\ Centre national de Recherches zootechniques, I.N.R.A., \\ 78350 Jouy en Josas
}

\section{RÉSUMÉ}

Sur des carcasses de porcs mâles entiers Large White abattus à 80 et roo $\mathrm{kg}$ de poids vif on a étudié les principales caractéristiques de la section transversale au niveau de la treizième côte et leurs liaisons avec d'autres critères d'appréciation de la composition de la carcasse (poids de la bardière, rapport longe/bardière, densité du " rein ").

I. Entre 80 et Ioo $\mathrm{kg}$, les mesures d'épaisseur du lard dorsal effectuées sur les sites latéraux, à $4,5 \mathrm{~cm}\left(\mathrm{P}_{1}\right)$ et à $6,5 \mathrm{~cm}\left(\mathrm{P}_{2}\right)$ de l'axe de la colonne vertébrale augmentent, de façon significative, respectivement de 15 et i 6 p. Ioo. Dans l'axe médian du corps (fente de la carcasse), l'épaisseur de lard n'augmente que de 7 p. Ioo.

L'accroissement de la surface de graisse sous-cutanée mesurée sur la section est de 22 p. roo et celui de la noix de côtelette de I6 p. Ioo.

2. La liaison entre la surface de graisse sous-cutanée et le poids de la bardière était, aux deux stades considérés, de $+0,86$ et $+0,82$, alors que les corrélations entre la surface de la noix de côtelette et le poids de la longe n'était, à 80 et Ioo $\mathrm{kg}$, que de $+0,58$.

Le rapport viande/graisse de la section (calculée d'après SIEBURG, 1957), s'est révélé fortement lié au rapport longe/bardière $(v=+0,9 \mathrm{I}$ et 0,90$)$, de même que l'indice de densité du rein $(r=+0,85$ et 0,89$)$.

L'application directe de ces résultats au contrôle des performances individuelles en station (performance-test) concerne la précision des méthodes d'amélioration génétique. 